\title{
Antiproliferative effects on tumor cells of the synthesized gold nanoparticles against Hep2 liver cancer cell line
}

\author{
Kamala Priya M R and Priya R. Iyer ${ }^{*}$ iD
}

\begin{abstract}
Background: Currently, nanomaterials (NMs) research and development is at a fastest pace due to the enhanced implication in different areas of applications. The synthesis of such NMs through biosynthesis methods is gaining much importance because of low cost involved and environment-friendly approach. In this present study, the nanoparticles (NPs) are synthesized using a medicinal plant, with anticancer properties so as to incorporate the therapeutic activity within the NPs, such that the NPs will have the attributes of NMs alongside the phytoactivity.

Results: The synthesis of gold nanoparticles (AuNPs) using Graviola, the fruit of Annona muricata (Ramaphal fruit) commonly referred as Mullu seetha fruit (Tamil), was successfully carried out. The initial confirmations of the NPs were using UV-vis spectra, which showed the characteristic peak for the NPs. HRSEM analysis gave an insight on the size and morphology of the NPs. The zeta potential was measured to check the stability of the NPs. The cytotoxicity was carried out in VERO cell line and anticancer study in Hep2 liver cancer cell line. The surface plasmon resonance (SPR) band showed the characteristic peak at $536 \mathrm{~nm}$ for the AuNPs. In SEM micrographs, the size was ranging between 20 and $30 \mathrm{~nm}$, on an average of $15 \mathrm{~nm}$ with spherical morphology. On the various tested concentrations in VERO cell line, the nanoparticles were non-toxic to the cells. The anticancer study gave an IC50 value at 10.94 $\mu \mathrm{g} / \mathrm{ml}$.
\end{abstract}

Conclusions: The NPs showed anticancer activity in treated Hep2 liver cancer cell line and as well as commendable non-toxic effect on normal VERO cell line. The results pose a positive impact to expand further studies in the development of potential drug molecules to tackle the disease of interest.

Keywords: Nanoparticles, HRSEM, Zeta potential, Cytotoxicity, Anticancer, Hep2 liver cancer cell line

\section{Background}

\section{Cancer-a major global public health issue}

Alarmingly 8.8 million people are dying each year of cancer, accounting to one out of six deaths and more than the number of deaths from HIV/AIDS, malaria, and tuberculosis combined. Particularly, in low-income and middle-income countries (LMICs), the disease management remains strenuous and the burden is greatest, where almost $75 \%$ of cancer deaths occur and the number of cancer cases is increasing steadily. It is estimated that cancer incidence will double by the year 2035 [1].

* Correspondence: brajuraj@yahoo.com

Post Graduate and Research Department of Biotechnology, Women's

Christian College, College Road, Chennai 600006, India
On an estimate, around $50 \%$ to $60 \%$ of patients will require radiotherapy at some time during their treatment; the International Atomic Energy Agency (IAEA) reported that in developing countries due to a shortage of 5000 machines, nearly $70 \%$ of cancer patients could not benefit from curative or palliative radiotherapy (International Atomic Energy Agency 2016). The IAEA are currently focusing to introduce radiotherapy to many of these countries. Moreover, it could be seen that investing in radiotherapy will save lives as well as have positive impact over economic benefits [2].

\section{Liver cancer-global context}

Liver cancer is the seventh most frequently occurring cancer in the world and the fourth most common cause of cancer mortality [3]. Its incidence rates have been 
gradually rising in many countries. The highest incidence rates of liver cancer in the world happen to be in Asia and Africa. A majority of cause lies within the chronic infections with hepatitis B virus (HBV) and hepatitis $C$ virus $(\mathrm{HCV})$ for almost three-quarters $(73.4 \%)$ of hepatic cellular carcinoma in the world [4].

\section{Barriers in current cancer therapy}

Current cancer therapy involves three major approaches such as surgical oncology, chemotherapy, and radiotherapy [5]. The major obstacles in the current anticancer agents are the development of drug resistance [6]. Multidrug resistance (MDR) is the simultaneous resistance to a number of structurally and functionally unrelated chemotherapeutic drugs. During cancer treatment, many cancers initially respond well to chemotherapy but subsequently lead to acquired resistance such that more than $90 \%$ of patients with metastatic cancer either fail to respond or relapse from chemotherapeutics. To address all these issues, various efforts have been invested to elucidate both on inherent and acquired MDR mechanisms [7].

\section{Inorganic compounds in cancer therapy}

Selenite is one of the most studied inorganic compounds because of its outstanding chemopreventive and anticancer features. It effectively inhibits cell proliferation of various types of cancer cells. Of different human cancer cell lines tested, lung cancer cells appeared to be more sensitive to selenite. A study reported that selenite cytotoxicity was correlated with Se uptake of three lung cancer cell lines (H157, H611, and U2020) and high concentrations (>1 mM) of selenate were non-toxic for these cell lines. Se plays a role as natural killer (NK) in cell-based anticancer immunotherapy where it increases the susceptibility of cancer cells to CD94/NK group, and has potential clinical applications in lung cancer patients. The combined effect of selenite and thioredoxin reductase inhibitors was detected in human ovarian and lung cancer cell lines. These results indicated the capacities of Se compounds to enhance the activity and reduce the toxicity of anticancer drugs. Additionally, these compounds appear to be more effective in inhibiting the growth of anticancer drug-resistant cancer cells compared with drug-sensitive cancer cells by means of deactivating various resistance mechanisms adapted by the cancer cells. Chemotherapeutic drug-resistant lung cancer cells were more sensitive to selenite compared to drug-sensitive cancer cells [8].

\section{Microorganisms in cancer treatment}

Microbes offer a feasible means of inducing anti-tumor immunity. Oncolytic viruses were among the first successfully used microbes in cancer therapy. An intratumoral injection of herpes virus, $\mathrm{T}$-VEC received approval from the
US Food and Drug Administration for metastatic melanoma in 2015. The authors used Escherichia coli, which are non-pathogenic, grow extracellularly, and persist for nearly 6 days after intratumoral injection. After the delivery of bacteria into the tumors, several rounds of bacterial lysis continues, a constant, high local concentration of CD47nb. It showed robust tumor-antigen-specific CD8 T cell responses that targeted non-injected tumors with established immunologic memory. These results strongly suggested the induction of adaptive immunity as the major therapeutic mechanism by which CD47 blockade operates in immune competent animals. Successful induction of $\mathrm{T}$ cell responses requires the presence of antigen as well as adjuvant to be present at the same time. The quorum-sensing bacteria achieve it through three major strategies. Initially, bacteria provide an abundance of adjuvant and persist at high levels for about a week in tumors. Followed by local delivery and quorum lysis prevent sepsis and leakage of therapeutics cargo into circulation, decreasing systemic exposure of the drug and generation of antidrug antibodies. And finally, bacteria have a remarkable ability for genetic cargo and can be explored further with more therapeutics in a strand [9].

\section{Cancer nanotechnology}

Nanoparticles (NPs) targeted for cancer therapies basically comprise of nanocarrier and the active drug, few NPs formulations of the drug by itself also exist. The nanodrug systems are advantageous with higher metabolic stability, enhanced membrane permeability, improved bioavailability, and prolonged activity. Nano transporters allow specialized targeted drug delivery of the anti-cancer agents to the tissue as well as at the cellular level. The key factors that affect the mucosal or transdermal absorption are size, surface charge, and hydrophobicity. The size of the particles plays a crucial role; smaller NPs are inducing higher transcellular uptake than the larger particles. But NPs larger than 300 $\mathrm{nm}$ could not be absorbed by intestinal cells. And only smaller NPs less than $500 \mathrm{~nm}$ can penetrate the bloodstream [10].

\section{Nanotechnology in biomedicine}

There are an increasing numbers of nanoparticle-based treatments being approved for clinical use which involves mostly spherical liposomes. However, scientists are constantly working on a wider range of NPs designs with different core materials bearing magnetic, optical, and biochemical properties and NPs of varying size, shape, hardness, and surface properties. Adding on to their therapeutic applications, NPs as well enable effective capabilities in imaging [11]. 


\section{Microbial-mediated synthesis of nanomaterials}

The microbial-mediated synthesis of nanomaterials (NMs) has been considered due to the following advantages like synthetized NMs have definitive chemical composition, size, and morphology; biosynthesis is carried out under at mild physicochemical conditions; ease of handling and cultivation of microbial cells; and possibility of tunable NMs with required characteristics by varying key parameters of cell culture set up. One of the greatest challenges faced in microbial nano biosynthesis is the control of dispersity of nanostructure materials, which primarily influence electronic and optical properties. When going for large-scale productions, costs of culture media for microbial growth affects to a greater extent. For instance, currently, bacterial nanocellulose applications are much limited to a few biomedical devices, mainly due to the costs of culture medium [12].

\section{Conventional chemical method of synthesis}

The synthesis of gold nanoparticles (AuNPs) was first reported in 1940 upon reacting gold chloride ( $\mathrm{HAuCl} 4)$ and trisodium citrate $(\mathrm{Na} 3 \mathrm{C} 6 \mathrm{H} 5 \mathrm{O} 7$ or $\mathrm{NaCt}$ ) which resulted in the formation of colloidal gold. Further on, the detailed work carried out by Turkevich and his coworkers has become one of the milestones of AuNPs synthesis. From then onwards, the method has been modified and improvised in order to meet the requirements of diverse area such as development of chemical sensors for water quality analysis using surface enhanced Raman spectroscopy (SERS), surface-induced catalytic activities, and drug delivery in biological systems and nano-toxicology studies. There are certain significant features of AuNPs that make them more attractive to employ in wide applications like surface plasmon resonance (SPR), a size- and shape-dependent property, and biocompatibility. Even though of all the distinctive properties of AuNPs, there is a major challenge residing over its usage, which is the control of the particle size and size distribution due to the fact of batch-to-batch variation affected by three factors, local temperature gradient, the efficiency of reagent mixing, and the resulting local concentration gradient [13].

\section{Green synthesis of nanomaterials}

Different physical and chemical methods such as solvothermal method, hydrothermal route, pulsed laser ablation technique, sonochemical process, and microwave irradiation has been exploited for the synthesis of NPs [14]. Conventional methods have limitations such as expensiveness, undesirable end products, and hazardous toxic chemicals, involving high temperature and pressure, etc. Whereas green synthesis is an environmentfriendly, cost-effective method to synthesize nanostructural material to have tunable structures, morphologies, and particle size distributions. Green synthesis methods employ organisms like plant, bacteria, fungi, etc. for the synthesis of NMs, and it has gained much interest in the rising area of research due to their less toxic or nontoxic nature, eco-friendly nature, and low cost of production. Plant extract are produced from leaves, stems, flower, and seeds of various plants. The extracts are rich in protein, amino acid, enzymes, vitamins, terpenoids, flavonoids, alkaloids, phenolic acids, etc., that act as capping and reducing agents that reduces metal ions during the bioreduction process to produce NPs or nanostructures in varied dimensions and morphologies [15].

\section{Limitations in the application of nanomaterials}

Nanotechnology has entered cancer therapy in the recent years. Upon then, many medical products have been approved for clinical use like albumin-boundpaclitaxel, liposomal doxorubicin, and liposomal irinotecan. At the moment, many anti-cancer nanodrugs are into various phases of clinical trials and preclinical research. But the major drawback is the increased cost of nanodrugs, compared with free drugs. Another limitation is the uptake of nanodrugs, which occurs through reticuloendothelial system (RES). Nanodrugs should avoid clearance through uptake by the (RES) which occurs primarily for particle size larger than $100 \mathrm{~nm}$. On the other aspect, localization of nanodrugs in tumorassociated macrophages will definitely increase the concentration of the drug at the tumor sites [10].

\section{AuNP's - as most preferred metallic nanoparticles}

Significantly, AuNPs have become versatile candidates used for cancer detection and therapy due to their futuristic physicochemical properties. But still, the use of toxic chemicals in developing AuNPs is of great concern since it may create environmental issues. To combat with this, biological methods of fabricating AuNPs are the much preferred choice. Hence, synthesis of AuNPs using plants, seaweeds, and microbes are advantageous over physical and chemical methods. On this context, the phytocomponents present in the natural source contribute to added bio-compatibility and cytotoxicity by stabilizing the AuNPs. There have been numerous studies stating on the anticancer properties of AuNPs synthesized through biological approach. More studies about the comparative evaluation of AuNPs against cancer and normal cells are needed for further extended research [16].

\section{Toxicity studies of metallic nanomaterials}

Though there are several advantages of metallic nanoparticles, there is much concern regarding their safety on biological cells as reported in in-vitro/in-vivo toxicity studies. In higher metallic form/bulk form, gold is inert/ 
non-toxic, but at the nano dimensions based on the manner in which these NPs are developed using different synthetic procedures and the nature of capping/stabilizing agents may lead to toxicity. Though green synthesized NPs using of various plant extracts containing polysaccharides, biomass, phytochemicals, and synthetic molecules are less toxic, there is a major drawback at the polydispersity, yield, purity, and metabolite formation aspects. There are many inconclusive data in the literature discussing whether AuNPs are toxic or nontoxic. The toxicity of NPs is also influenced by the size, shape, and surface chemistry of NPs. Some NPs have demonstrated size-dependent toxicity, whereas others have shown surface chemistry-dependent toxicity. Therefore, predicting toxicity becomes difficult since similar sizes of NPs results in differential toxicity on the same cell line [17].

\section{Anticancer effects of Annona muricata's Fruit-Graviola} Annona muricata is a tropical tree widely distributed in the regions of Africa, South America, and Southeast Asia. It belongs to the family Annonaceae. It is commonly referred as soursop, graviola, and guanabana. The fruit is edible in green heart-shaped and the tree comprises of large, glossy, dark green leaves. It has been reported in the literature that nearly 74 bioactive compounds of the plant exhibit a variety of anticancer effects in in vitro and in vivo animal model systems. $A$. muricata-derived preparations and formulations have been used for the treatment of different disease conditions, thereby proving its prominence in Ethnomedicine [18]. The bark, leaves, and roots of Annona muricata have been extensively applied globally in the disease management of various ailments, including inflammatory conditions, rheumatism, diabetes, hypertension, insomnia, cystitis, parasitic infections, and cancer [19].

\section{Traditional medicines - treatment of liver disorders}

Herbal and traditional medicines have been used to treat liver disorders for centuries. A. muricata is commonly used to treat various liver disorders, particularly jaundice. In a recent study, performed on phenyl hydrazineinduced jaundice in adult rats, treated with $A$. muricata aqueous extract at different doses, found a substantial reduction in elevated bilirubin. It has also been reported to protect against increased serum glutamic-oxaloacetic transaminase (SGOT), serum glutamic-pyruvic transaminase (SGPT), alkaline phosphatase (ALP), and liver and brain lipid peroxidation. In addition to the hepatoprotective properties, the aqueous extract was reported to protect against liver damage induced by $\mathrm{CCl} 4$ and acetaminophen. The glucosides of $A$. muricata mechanistically reduced the bilirubin levels by converting to glucuronic acid that conjugates with bilirubin for excretion.
Apparently, the phytochemicals in the extract may act as regulators increasing the activity of enzymes and transporters, and thereby enhancing the overall activity involved in clearing bilirubin. Combining all these outcomes, the potential hepato-protective function $A$. muricata is clearly emphasized and its prominent role in liver function [19].

Hence, A. muricata was used for the synthesis of AuNPs in this study. Further, the synthesized nanoparticles were characterized and checked for the in vitro cytotoxicity in VERO cell line and anticancer activity in Hep2 liver cancer cell line.

\section{Methods}

\section{Synthesis of the AuNPs}

\section{Preparation of gold chloride solution}

Gold chloride was used in the synthesis of nanogold particles. Chloroauric solution was prepared by dissolving required volumes of $\mathrm{HAuCl} 4$ in $1000 \mathrm{ml}$ of deionized double-distilled water. A stock solution of required molar concentration was prepared and from this the required working solution was used for the synthesis. The working solution was adjusted for the required $\mathrm{pH}$ and used as such without any further dilutions for the synthesis [20].

\section{Preparation of the plant extract}

The fruit of Annona muricata, Graviola (Soursop) was brought from the local fruit store in Purasaiwalkam, Chennai, India. The formal identification of the plant material used in the study was identified by Dr. G. Jeya Jothi, Taxonomist, Department of Plant Biology \& Biotechnology, Loyola College, Chennai. I would like to confirm that no voucher specimen of this material has been deposited. About $5 \mathrm{~g}$ of the peel/pulp were taken and washed repeatedly to remove the soil and dirt over them. Then, $50 \mathrm{ml}$ of double-distilled water was added to the finely cut leaves and taken in a conical flask. The flask was heated for 10-15 min on flame. Then, it was filtered and the extract was collected. The extract was stored at $4{ }^{\circ} \mathrm{C}$ and used for the further synthesis [21].

For the synthesis of AuNPs, the $\mathrm{pH}$ of the stock solution was checked and adjusted. To the required quantity of chloroauric acid solution, about $1 \mathrm{ml}$ of plant extract was added. The plant extract served as the reducing and stabilizing agent for the synthesis. The pale yellow color of the chloroauric acid changed into a wine red, pink or purple color, confirming the formation of nanoparticle.

\section{Characterization of nanoparticles using UV-vis spectra}

The bioreduction of gold chloride in aqueous solution was monitored by periodic sampling of the aliquots of the suspension. One milliliter of the sample was diluted with $30 \mathrm{ml}$ of double-distilled water and analyzed for the 
presence of nanoparticles. The UV-vis spectra of the AuNP's were recorded using a Systronic smart DBS 2303 UV-vis spectra operating in the wavelength of 400 to $700 \mathrm{~nm}$.

\section{HRSEM analysis}

The NPs were diluted with Millipore water in the ratio of 1:20 such that the concentration of the NPs became much less, thus enabling better imaging of the NPs. About $10 \mu \mathrm{l}$ of sample was placed on the aluminum foil and allowed to air dry. Then the scanning electron micrographs of the samples was carried out at SAIF (Sophisticated Analytical Instrumentation Facility), IIT Madras, Adyar [22].

\section{Zeta potential}

The stability of the NPs synthesized is elucidated on analysis of zeta potential. It is the measure of the charged species present at the NPs surface that prevents from aggregation and ensuring highly stable nanoformulations. The more the positive or the more the negative, the zeta measure possess the strongest stability.

\section{Fourier-transform infrared spectroscopy analysis}

The Fourier-transform infrared spectroscopy (FTIR) analysis method uses infrared light to scan the test samples and observe its chemical properties and other constituents. The synthesized NPs were analyzed for the presence of functional groups of plants that aided in the process of NPs formation. The study was performed using the FT-IR Spectrophotometer (Perkin Elmer) at Bharathidasan University, Trichy.

\section{Phytochemical analysis of the green extracts of the plants tested}

The plant extracts were tested for various phytochemicals such as terpenoids, flavonoids, saponins, tannins, phenols, Coumarins, Triterpenoids, and the results were recorded. These phytochemicals act as the reducing and stabilizing agents for the plant mediated synthesis of nanoparticles.

\section{In vitro cytotoxicity of the AuNPs \\ Procedure}

The VERO cell line was used to check cytotoxicity of the synthesized AuNPs. The cell line used was procured from Translational Research Platform for Veterinary Biologicals (TRPVB) TANUVAS, Chennai. About $1 \times 10^{4}$ cells $(10$, 000 total cells) were added into each well of 96 -well plates and incubated overnight. To this, different concentration of AuNPs was added. The treated cells were incubated for $24 \mathrm{~h}$ at $37^{\circ} \mathrm{C}$ in the $\mathrm{CO}_{2}$ incubator. About $10 \mu \mathrm{l}(5 \mathrm{mg} /$ $\mathrm{ml}$ of 3-(4,5-dimethylthiazol-2-yl)-2,5-diphenyltetrazolium bromide (MTT) was added to each well. The cells were incubated for about 3 hours for the formation of formazan crystals. About $100 \mu \mathrm{l}$ of dimethyl sulfoxide (DMSO) solvent was added to dissolve the formazan crystals and incubated for about an hour. The absorbance was read at $540 \mathrm{~nm}$ with a reference filter of $620 \mathrm{~nm}$. Percentage of cell viability was calculated. Formula for cell inhibition $=($ O.D of treated cells $/$ O.D of control $) \times 100$. Formula to check cell viability $=(100-$ cell inhibition $)$

\section{Anticancer activity of the AuNPs Procedure}

The Hep2 liver cancer cell line was used to check antiproliferative activity of the synthesized nanoparticles. The cell line used was procured from Kings Institute, Guindy, Chennai. About $1 \times 10^{4}$ cells $(10,000$ total cells) were added into each well of 96-well plates and incubated overnight. To this, different concentration of AuNPs was added. The treated cells were incubated for $24 \mathrm{~h}$ at $37{ }^{\circ} \mathrm{C}$ in the $\mathrm{CO}_{2}$ incubator [6]. About $10 \mu \mathrm{l}(5 \mathrm{mg} / \mathrm{ml})$ of MTT was added to each well. The cells were incubated for about $3 \mathrm{~h}$ for the formation of formazan crystals. About $100 \mu \mathrm{l}$ of DMSO solvent was added to dissolve the formazan crystals and incubated for about an hour. The absorbance was read at $540 \mathrm{~nm}$ with a reference filter of $620 \mathrm{~nm}$. Percentage of cell viability was calculated. Formula for cell inhibition $=(\mathrm{O} . \mathrm{D}$ of treated cells $/$ O.D of control $) \times 100$. Formula to check cell viability $=$ (100 - cell inhibition) [23].
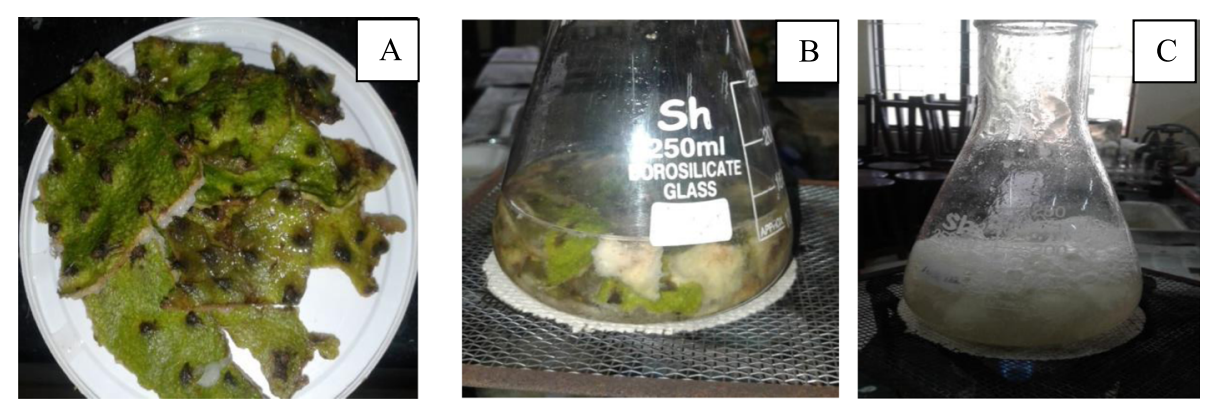

Fig 1 a-c Extracts of Graviola peel and pulp, preparation for the pulp extract 


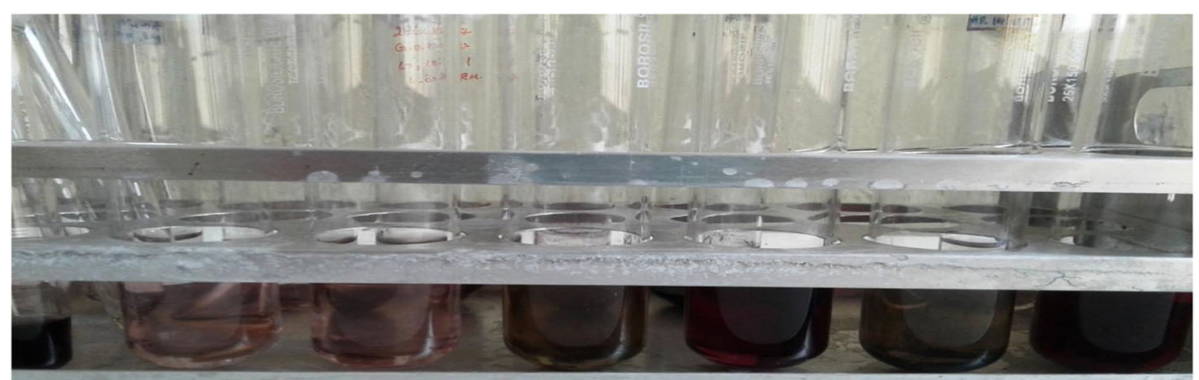

Fig. 2 The synthesized AuNPs using A. muricata peel and pulp extract

\section{Results}

\section{Synthesis of AuNPs}

The NPs were synthesized using the pulp and peel extract of the soursop fruit, Annona muricata on reduction with chloroauric acid. The synthesized NPs were pale pink to deep wine red in colour (Figs. 1 and 2).

\section{Effect of $\mathrm{pH}$ on the synthesis}

The $\mathrm{pH}$ has a prominent role in the synthesis of the NPs. A pH range of $\mathrm{pH} \mathrm{6,} \mathrm{pH} 8$, and $\mathrm{pH} 10$ were tested. It was found that $\mathrm{pH} 8$ to be the optimum $\mathrm{pH}$ for the synthesis of NPs. At other $\mathrm{pH}$ gradients, the reduction of chloroauric acid was not complete; a partial reduction was noted from the colour changes of the chloroauric solution [24].

\section{UV Visible Spectrophotometry}

The UV absorption peak is the respective wavelength at which the optical absorption spectra of metal nanoparticles are measured by the surface plasmon resonance. There exists a correlation between the wavelength and the particle size, wherein wavelength shifts to longer spectrum with increasing particle size. Based on the particle size, the position and shape of plasmon absorption of AuNPs highly varies. The initial confirmation of the NPs synthesis was through UV absorption spectrum, which was showing characteristic peaks for the synthesized AuNPs.

Similar results has been stated for nanoparticles synthesis by Jae Yong Song et al. 2009 (Fig. 3).

\section{Zeta potential}

The stability of the synthesized NPs were measured through zeta potential analysis. The zeta potential analysis the associated charges present at the surface of the NPs. This charge aids much more in the higher stability of the NMs. It helps the particles to stay monodispersed and prevents agglomerations. The zeta measures exist in polar ranges, it is either toward positivity or negativity. Both of these ranges assure high stability of the NPs. The zeta potential measurement also gives projection over the stability of the synthesized colloidal suspensions. In general, when the zeta potential value is about $>30 \mathrm{mV}$, there are less chances for agglomeration of the particles due to the electrostatic forces between the particles (Devika R. Bhumkar et al. 2007). The results observed were in concordance to similar reports by Devika R. Bhumkar et al. 2007 (Fig. 4).
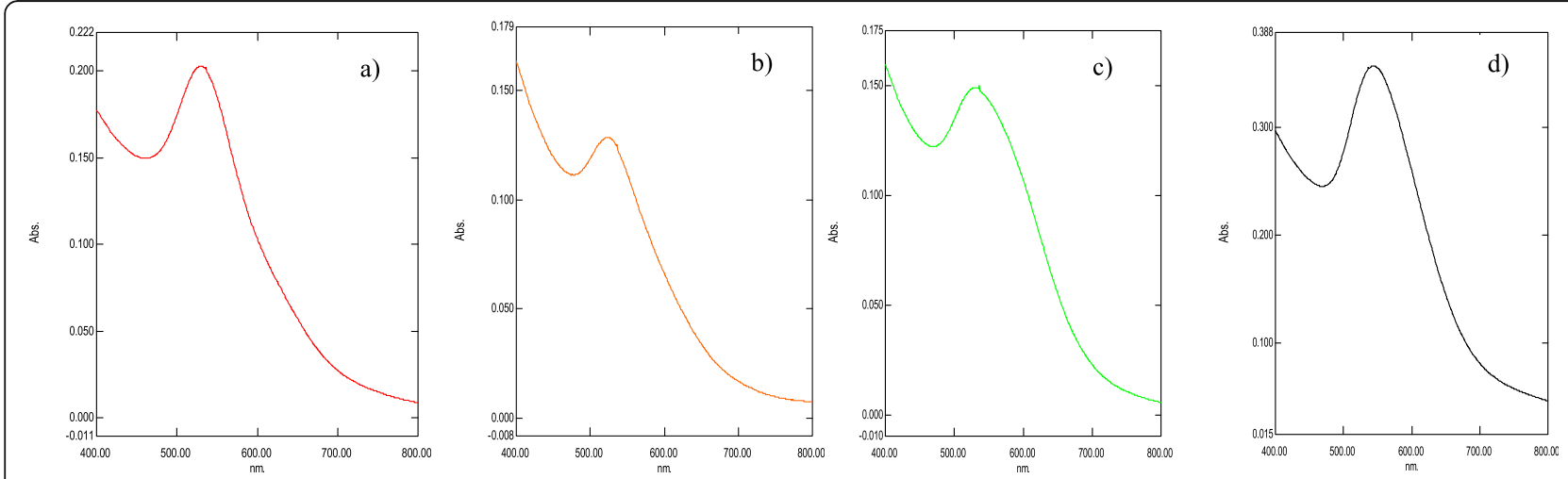

Fig. 3 a-d UV absorbance spectrum of AuNPs synthesized by A. muricata peel at $10^{-1}$ ( $\left.\lambda_{\max }-529 \mathrm{~nm}\right)$, A. muricata pulp at $10^{-1}\left(\lambda_{\max }-525 \mathrm{~nm}\right)$, A. muricata peel at $10^{-3}\left(\lambda_{\max }-520 \mathrm{~nm}\right)$, A. muricata pulp at $10^{-3}\left(\lambda_{\max }-543 \mathrm{~nm}\right)$. The peak confirms the formation of AuNPs 


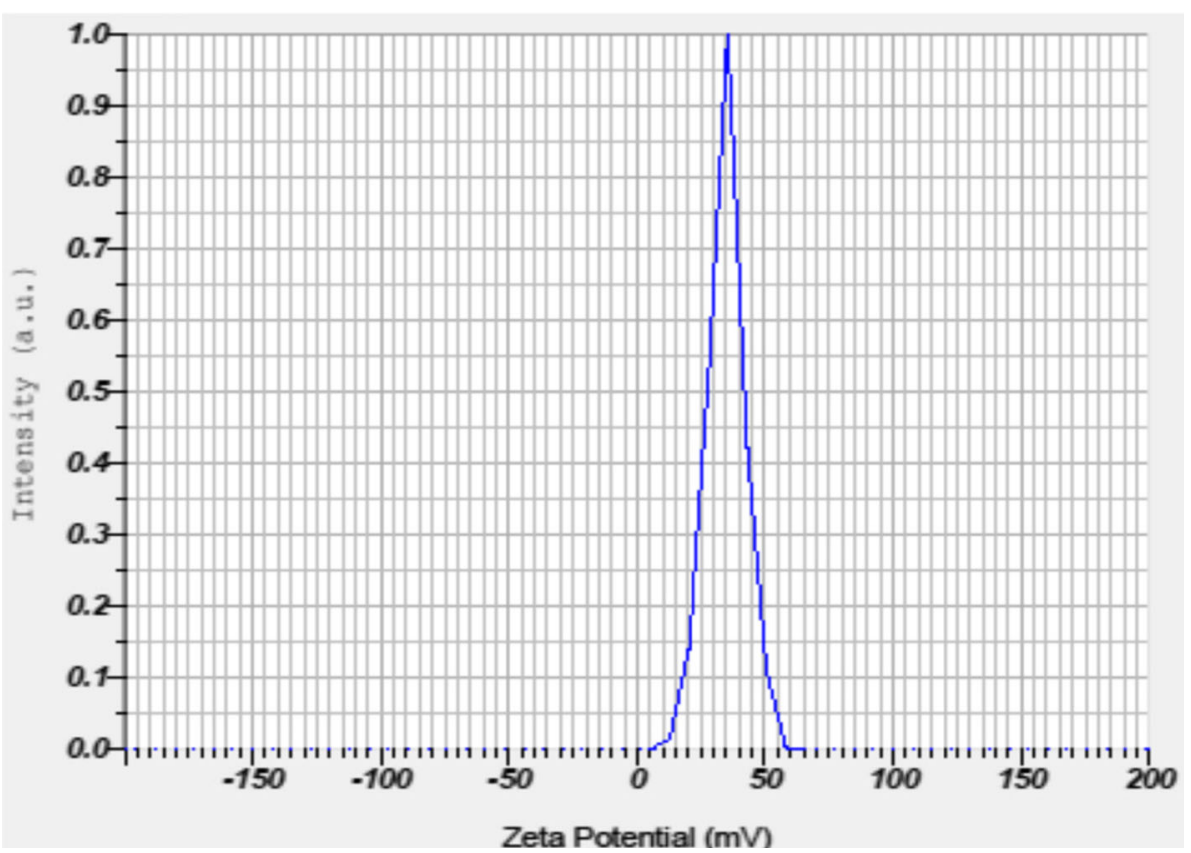

Fig. 4 Zeta potential analysis. Zeta potential (mean): - $30.9 \mathrm{mV}$. Zeta potential (mean): $-32.9 \mathrm{mV}$. Zeta potential (mean): $-34.9 \mathrm{mV}$

\section{HRSEM analysis}

The size of the NPs was $12 \mathrm{~nm}$ upwards, and an average about $20 \mathrm{~nm}$; a commendable nano dimension achieved through plant-mediated synthesis. The NPs were spherical in morphology and evenly distributed. The significance on the size of the nanoparticles has been similarly stated by Jayanth and Vinod 2002 (Fig. 5).

\section{FT-IR analysis}

\section{FT-IR analysis of $A$. muricata peel}

Table 1 shows the FT-IR analysis of $A$. muricata peel.

Table 1 FT-IR analysis A. muricata peel

\begin{tabular}{lll}
\hline S. No. & Wave number (absorptions) $\left(\mathrm{cm}^{-1}\right)$ & Functional groups \\
\hline 1. & 1384.25 & $(-\mathrm{C}-\mathrm{H})$ alkane \\
2. & 1416.38 & $(\mathrm{C}=\mathrm{C})$ aromatic \\
3. & 1641.75 & $(\mathrm{C}=\mathrm{C})$ Alkene \\
\hline
\end{tabular}

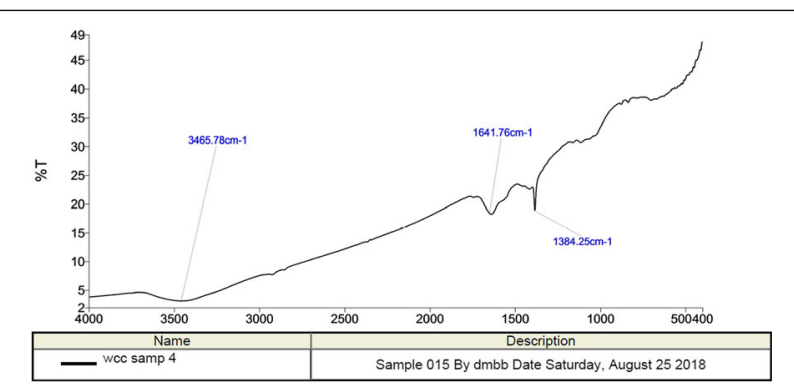

\section{FT-IR analysis of A. muricata pulp}

Table 2 shows the FT-IR analysis of $A$. muricata pulp.

The FT-IR spectra of organic compounds have two general areas, the functional group region (4000-1500 $\mathrm{cm}^{-1}$ ) and the finger print region $\left(1500-400 \mathrm{~cm}^{-1}\right)$. The peaks present in the functional group region correspond to the particular type of bonds present in the compound and hence can be used to identify the specific functional

Table 2 FT-IR analysis A. muricata pulp

\begin{tabular}{lll}
\hline S. No. & Wave number (absorptions) $\left(\mathrm{cm}^{-1}\right)$ & Functional groups \\
\hline 1. & 414.55 & \\
2. & 490.38 & \\
3. & 620.91 & $=(-\mathrm{C}-\mathrm{H})$ alkane \\
4. & 1113.78 & $(\mathrm{C}-\mathrm{O})$ alcohol, $(\mathrm{C}-\mathrm{O})$ Ester \\
5. & 1384.23 & $(-\mathrm{C}-\mathrm{H})$ alkane \\
6. & 1639.72 & $(\mathrm{C}=\mathrm{C})$ Alkene \\
7. & 3465.22 & $(\mathrm{~N}-\mathrm{H})$ Amine, $(\mathrm{O}-\mathrm{H})$ alcohol \\
\hline
\end{tabular}

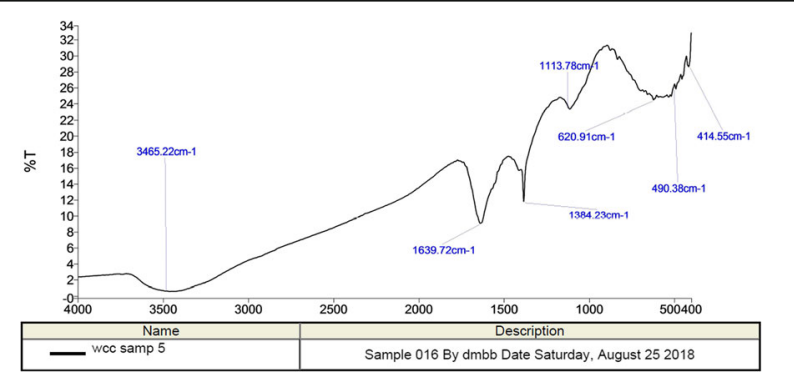




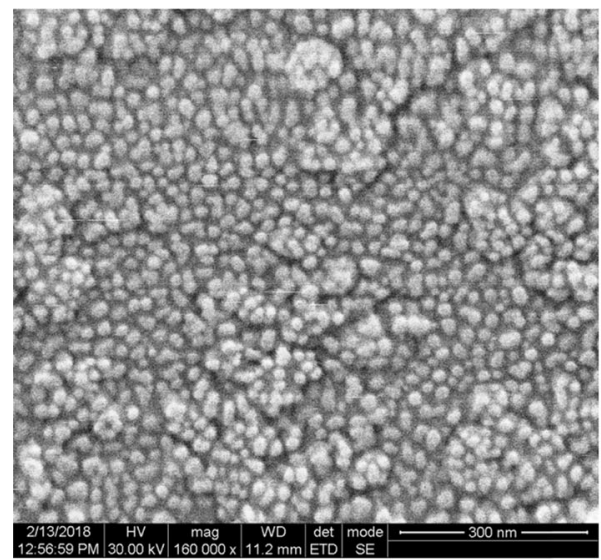

a

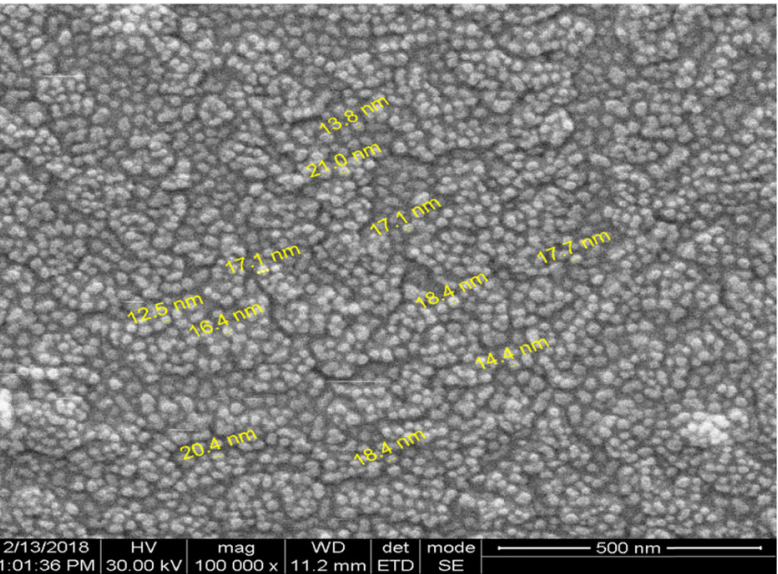

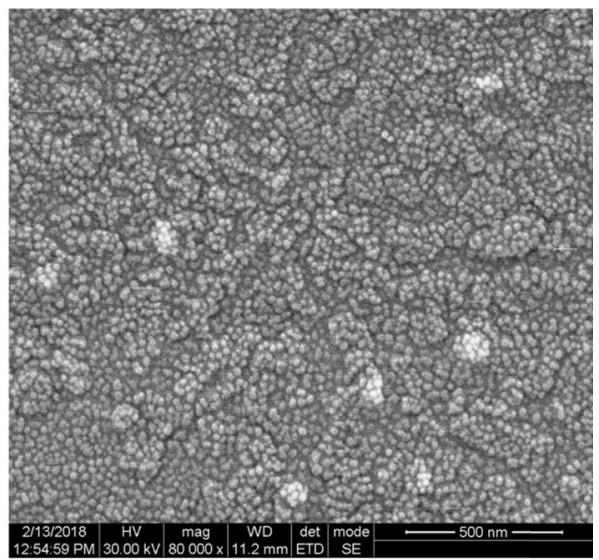

$\mathrm{b}$

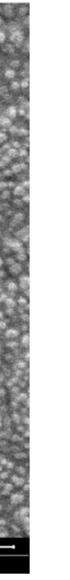

(

Fig. 5 a HRSEM images of the nanoparticles. b Nanoparticles showing spherical morphology. c Size of the nanoparticles measured

groups of the compound under study. The peaks present in the fingerprint region are a result of complex deformations of the molecule. In particular, they may be a characteristic of molecular symmetry or combination of bonds deforming simultaneously. Similar results have been reported by Jolly Mariam Johny et al. (2011).

\section{Phytochemical analysis of the green extracts of the samples used}

The phytochemistry of the plant extracts were carried out to check for the various secondary metabolites and constituents of the plant. The results showed the presence of many compounds as listed below (Table 3).

\section{In vitro cytotoxicity in VERO cell line}

The in vitro cytotoxicity assay was carried out in VERO cell line. The results obtained show that the AuNPs are having higher cell viability under all the various tested concentrations. Thereby the synthesized AuNPs are non-toxic over the treated VERO cell line.
The viability \% of the AuNPs was illustrated through MTT assay in the VERO cell line, which showed that the AuNPs under study has good cell viability percentage at all the tested concentrations. Hence the synthesized AuNPs can be considered for further extended study (Fig. 6).

Table 3 The various phyto chemicals present in the plant

\begin{tabular}{llll} 
extract & & & \\
\hline S. No. & Compounds & A. muricata peel & A. muricata pulp \\
\hline 1. & Coumarins & + & + \\
2. & Saponins & + & + \\
3. & Flavanoids & - & - \\
4. & Terpenoids & + & + \\
5. & Triterpenoids & - & - \\
6. & Carbohydrates & + & + \\
7. & Phenols & Yellow color & Yellow color \\
8. & Tannins & + & + \\
9. & Alkaloids & + & + \\
\hline
\end{tabular}

$+=$ Presence of the compound, $-=$ absence of the compound

The number of signs indicates the intensity of results observed 
In vitro anticancer activity in liver cancer Hep2 cell line The results of the anticancer activity on the liver cancer Hep2 cell line showed that the Graviola mediated synthesized AuNPs, using pulp and peel, have demonstrated well antiproliferative activity against the cancer cell line. And proved to be efficient in most of the concentrations tested and the inhibition concentration 50 IC50 was at $17.98 \mu \mathrm{g} / \mathrm{ml}$ for NP1 and $13.08 \mu \mathrm{g} / \mathrm{ml}$ for NP 2 recorded out of the study. Based on the study, the AuNPs synthesized using Graviola peel extract has higher anticancer activity (Fig. 7).

Comparative anticancer activity between NP 1 \& NP 2
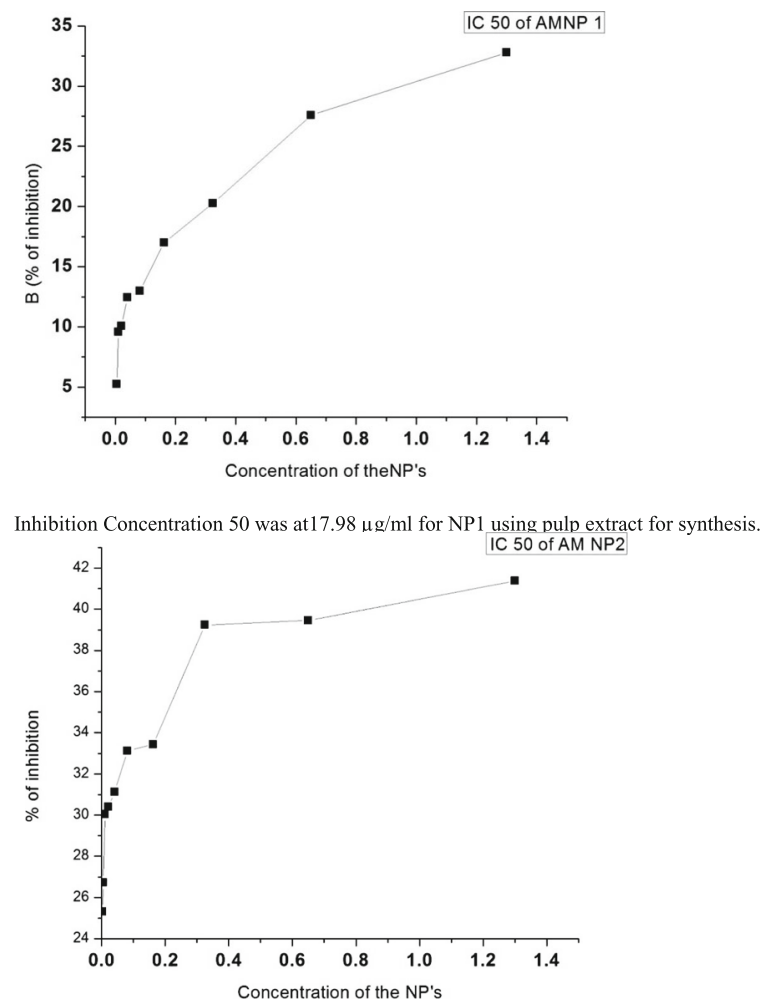

Inhibition Concentration 50 was at $13.08 \mu \mathrm{g} / \mathrm{ml}$ for NP2 using pulp extract for synthesis.

\section{Fluorescence assisted cell sorting-FACS analysis Treated VERO cells}
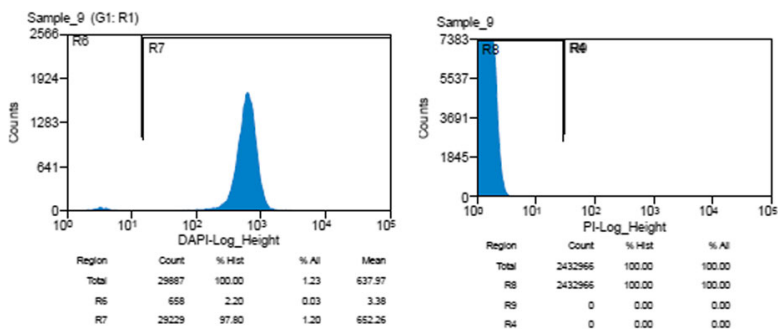

\section{Treated Hep2 cells}
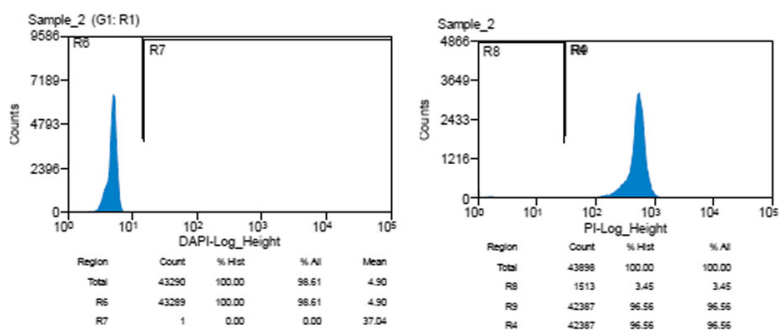

Fluorescence activated cell sorting (FACS) differentiates between the live and dead cells in the given population. The cells were labeled with PI (propidium iodide) and DAPI (4',6-diamidino-2-phenylindole). DAPI was taken up by live cells, whereas PI stained the dead cells. In treated VERO cells, the percentage of live cells were highest and in treated Hep2 cells the PI log was seen in highest percentage. The results were much in concordance to the in vitro MTT assay. Similar analysis has been reported by Chih-Jen Lai, et al. 2019 [25].

\section{Discussion}

Ethnomedicine is emerging again from its dormancy. The traditional use of plants as therapeutic agents is an age old practice in many countries and cultures [26]. The results from the study imply a successful implication of an ethnomedically important plant for the treatment of cancer. The Graviola fruit is widely known for its anticancer activity as a crude extract. The present study has exploited the plant in the synthesis of nanoparticles and the anticancer activity of the synthesized nanoparticles is well demonstrated. Further, the bioactive compounds that are underlying this activity have to be elucidated. The extraction and purification of those bioactive compounds will help us to understand the mechanism of action against the disease. The anticancer studies can be extended targeting other types of cancer [27].

For a better oncotherapy and tumor targeting, nanotechnology-based drug delivery systems provides an exceptional opportunity. Among the various available nanotechnology-based strategies for tumor targeting, nanocrystals play a remarkable role since they are highly suitable for passive and active targeting with maximum possible drug content almost to $100 \%$. In nanotechnologybased systems, there is a possibility to deliver together diagnostic probes along with the anticancer drugs. This combinational tumor theranostics approach results in the treatment of cancer, in a way simultaneously recognition, its evaluation, and transmission stages of the tumor. The multiple nanotechnology-based delivery systems utilized for tumor theranostics are polymeric nanoparticles, liposomes, micelles, polymersomes, etc. [28]. 


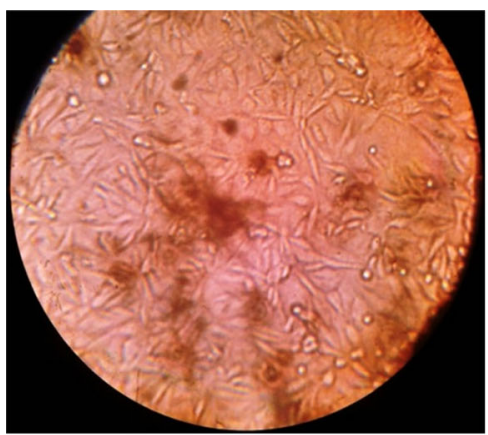

a

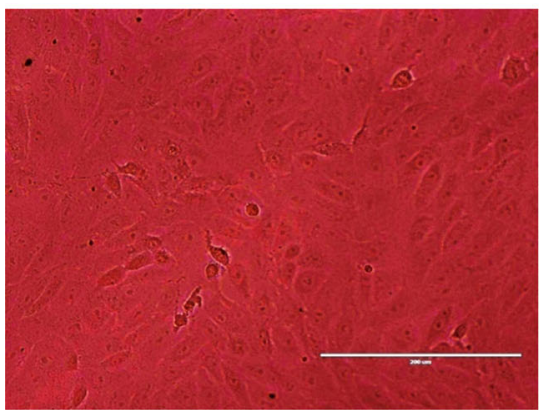

C

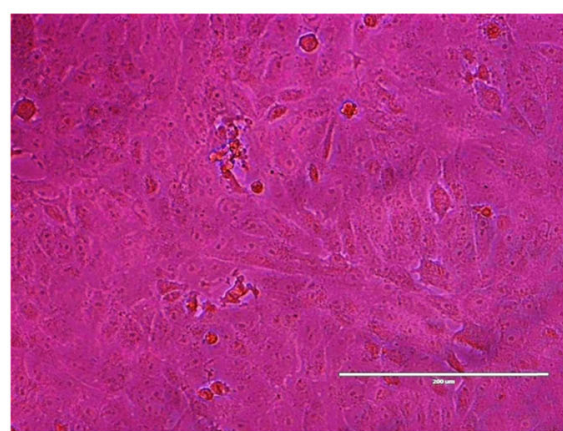

b

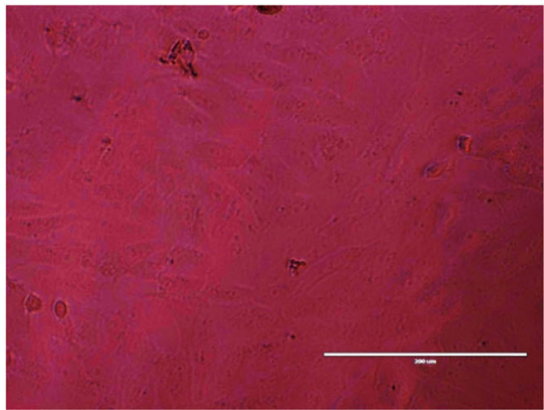

$\mathrm{d}$

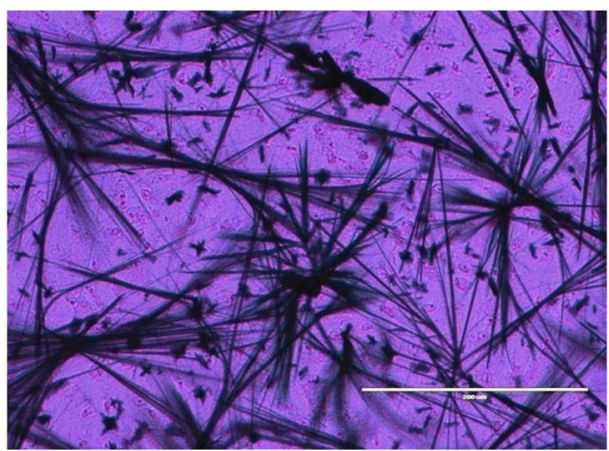

e

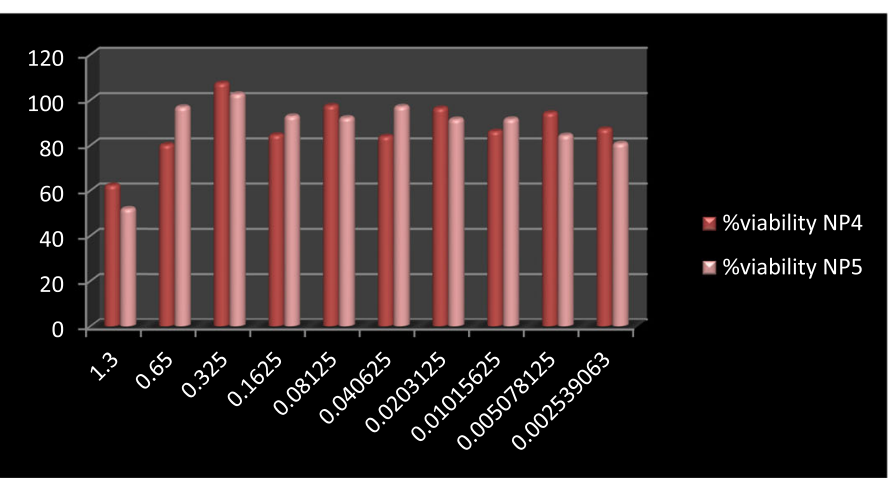

$\mathrm{f}$

Fig. 6 a VERO cell line. b Control cell after 24 h. c After treated with AMNP1. d After treated with AMNP2. e The formazan crystals formed in treated VERO cells followed by MTT dye incubation. $\mathbf{f}$ Viability \% in treated VERO cell line 


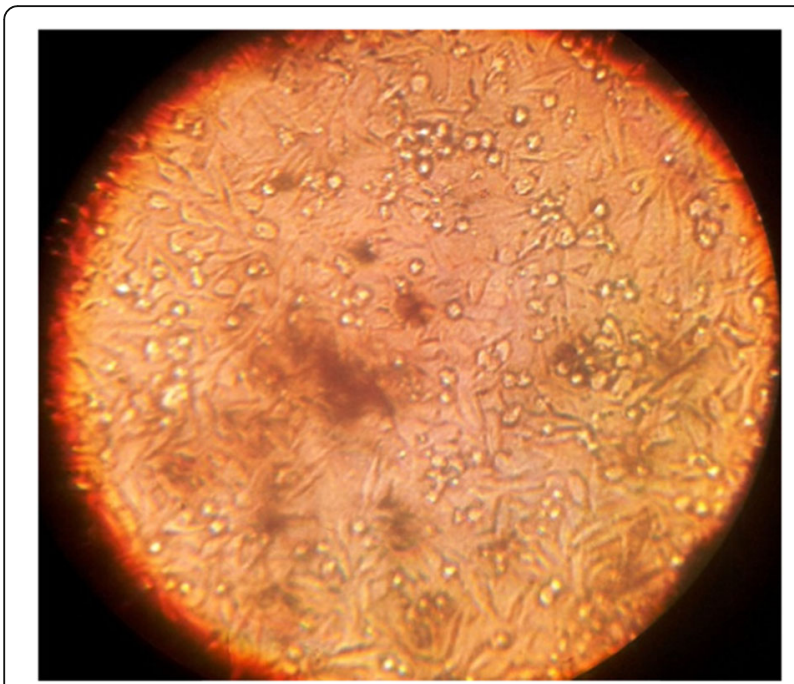

Fig. 7 a Hep2 liver cancer cell line

Presently, NPs have been extensively made use of topical drug delivery for wound healing. AuNPs have been much exploited for their potential antibacterial properties. Different types of nanocarriers have been used to enhance the permeation of drugs through skin as an alternative route to overcome the challenges of conventional oral and parenteral routes. Additionally to the use of lipid-based nanocarriers for dermal application of drugs, some polymeric nanocarriers showed an equally promising role in their places. The nanocapsules were used to deliver the antiandrogenic drug flutamide to the hair follicles thus providing a controlled and sustained drug release and thereby minimized penetration to the systemic circulation. Supportively, confocal microscopy imaging showed the follicular localization of the fluorescently labeled nanocapsules (NCs). To achieve long-term stability, NPs suspension can be converted to dry powder nanocomposites by spray-drying with inert carriers [4].

\section{Conclusion}

Currently, ethnomedicine is gaining its past glory and prominently being utilized at various treatments and therapies. On this context, the therapeutic properties pertaining to one of the medicinal plants, Annona muricata, was exploited in this study. The synthesis of AuNPs using pulp and peel extracts of the fruit of $A$. muricata, Soursop fruit was carried out successfully. The characterization of the synthesized nanoparticles was analyzed under UV-vis spectra, zeta potential, HRSEM, FTIR, and phytochemistry. The in vitro cytotoxicity showed that the synthesized gold NP's are nontoxic over VERO cell line and anticancer studies against Hep2 cell line proved that the NP's have good antiproliferative effect over the liver cancer cell line. Comparatively, the AuNPs mediated by peel extract have higher anticancer activity than AuNPs mediated by pulp extract. Finally, FACS analysis was performed to determine the live and dead cell populations in support of the in vitro results, which proved that the in vitro results were in concordance with the FACS analysis.

\section{Abbreviations}

AuNPs: Gold nanoparticles; DMSO: Dimethyl sulfoxide; FACS: Fluorescence assisted cell sorting; FTIR: Fourier-transform infrared spectroscopy analysis; HRSEM analysis: High-resolution scanning electron microscope; MNPs: Metallic nanoparticles; MTT: 3-(4,5-Dimethylthiazol-2-yl)-2,5diphenyltetrazolium bromide; NMs: Nanomaterials; NPs: Nanoparticle

\section{Acknowledgments}

I would like to acknowledge Dr. Sathish Kota, Scientist, TRPVB, TANUVAS, Chennai and Dr. Harini Challa, Post-Doctoral Fellow, TRPVB, TANUVAS, Chennai for their courteous assistance all through the in vitro studies.

\section{Authors' contributions}

$\mathrm{PI}$ designed and directed the study and KP executed the entire research work from synthesis of NPs, its characterization, phytochemical analysis, in vitro studies, and was the major contributor in writing the manuscript Both authors read and approved the final manuscript.

\section{Funding}

This work was funded by the TNSCST (TamilNadu State Council for Science and Technology) through RFRS (Research Funding for Research Scholars) (2016-2018)

\section{Availability of data and materials}

Not applicable.

\section{Ethics approval and consent to participate} Not applicable.

\section{Consent for publication}

Not applicable.

\section{Competing interests}

The authors declare that they have no competing interest.

Received: 5 December 2019 Accepted: 23 January 2020

Published online: 06 March 2020

\section{References}

1. Prager GW, Braga S, Bystricky B et al (2018) Global cancer control: responding to the growing burden, rising costs and inequalities in access. EMSO. 3:1-10

2. Olver I et al (2017) Cancer control—a global perspective. European Journal of Cancer Care 26(1):1-6

3. Jessica L. Petrick et al., 2019, The changing epidemiology of primary liver cancer. Current Epidemiology Reports 6, 104-111

4. Elzoghby AO (2019) Pharmaceutical nanotechnology in Egypt: diverse applications and promising outcomes. Nanomedicine. 1-6

5. Nagajyothi PC et al (2017) Green synthesis: in-vitro anticancer activity of copper oxide nanoparticles against human cervical carcinoma cells. Arab J Chem 10:215-225

6. Zhai Lu et al., 2017, Experimental study on the apoptosis of cervical cancer Hela cells induced by juglone through c-Jun N-terminal kinase/c-Jun pathway. Asian pacific journal of tropical medicine1-4

7. Arnst KE, Banerjee S, Chen H et al (2019) Current advances of tubulin inhibitors as dual acting small molecules for cancer therapy. Med Res Rev 1-29

8. Tan HW et al (2018) Selenium species: current status and potentials in cancer prevention and therapy. Int J Mol Sci 20(75):1-26

9. Dougan M, Dougan SK (2019) Programmable bacteria as cancer therapy. Nat Med 25:1030-1036

10. Kopeckovaa K et al (2019) Nanodrugs used in cancer therapy. Biomed Pap Med Fac Univ Palacky Olomouc Czech Repub 163(2):122-131

11. Grodzinski P et al (2019) Integrating nanotechnology into cancer care. ACS Nano 13:7370-7376 
12. Grasso G et al (2019) Microbial nanotechnology: challenges and prospects for green biocatalytic synthesis of nanoscale materials for sensoristic and biomedical applications. Nanomaterials 2020(10):11

13. Dong J et al (2019) Synthesis of precision gold nanoparticles using Turkevich method. KONA Powder and Particle Journal 1-9

14. Govindaraju K et al (2019) Seaweed (Turbinaria ornata)-assisted green synthesis of magnesium hydroxide $[\mathrm{Mg}(\mathrm{OH}) 2]$ nanomaterials and their antimycobacterial activity. Mater Chem Phys 239:1-4

15. Surya Pratap Goutam et al., 2019, Green synthesis of nanoparticles and their applications in water and wastewater treatment. Bioremediation of Industrial Waste for Environmental Safety, 349-379.

16. Jeyarani $S$ et al (2019) Biomimetic gold nanoparticles for its cytotoxicity and biocompatibilityevidenced by fluorescence-based assays in cancer (MDA-MB231) and non-cancerous (HEK-293) cells. J Photochem Photobiol B Biol 202:1-6

17. Akash Kumar et al., 2019, A novel approach towards synthesis and characterization of non-cytotoxic gold nanoparticles using taurine as capping agent. Nanomaterials 2020, 10, 45, 1-19

18. Rady I et al (2018) Anticancer properties of Graviola (Annona muricata): a comprehensive mechanistic review, oxidative medicine and cellular longevity. 2018:1-39

19. Asif Khurshid Qazi et al., 2018, Emerging Therapeutic Potential of Graviola and its Constituents in Cancers, https://academic.oup.com/carcin/advancearticle abstract/doi/10.1093/carcin/bgy024/4859483

20. Kamala Priya MR, Iyer PR (2014) Extracellular rapid biosynthesis of AuNP's using various green extracts of plants. International journal of molecular biology \& biochemistry 2:33-40

21. Kamala Priya MR, lyer PR (2019) Anti-cancer study in hela cell line of phytosynthesized gold nanocompounds using Andrographis paniculata (nilavembu) — characterisation, optimization, phytochemical analysis \& anti microbial study. Indian Journal of Applied Research 9:24-27

22. Kamala Priya MR, lyer PR (2015) Comparitive study on the characterisation of AuNP's synthesized using various plant extracts. International Journal of Phytotherapy 5:17-21

23. M R Kamala Priya \& Priya R. lyer. 2014, Anticancer studies of the synthesized AuNP's against MCF 7 breast cancer cell line. Applied Nanoscience 443-448

24. Kamala Priya MR, lyer PR (2014) Studies on the various parameters underlying the Synthesis of nanoparticles and the in-vitro stability of nanoparticles. Frontiers in Biotechnology 1-4

25. Lai C-J et al (2019) CD44 Promotes migration and invasion of docetaxelresistant prostate cancer cells likely via induction of hippo-yap signaling. Cells 8(295):1-13

26. Li J et al (2008) queous extract of Solanum nigrum inhibit growth of cervical carcinoma (U14) via modulating immune response of tumor bearing mice and inducing apoptosis of tumor cells. Fitoterapia 79:548-556

27. Kamala Priya MR, Iyer PR (2015) Applications of the green synthesized AuNP's-antimicrobial activity, water purification system and drug delivery system. Symbiosis 1-4

28. Joseph E, Singhvi G et al (2019) Multifunctional nanocrystals for cancer therapy: a potential nanocarrier. Nanomaterials for Drug Delivery and Therapy $91-116$

\section{Publisher's Note}

Springer Nature remains neutral with regard to jurisdictional claims in published maps and institutional affiliations.

\section{Submit your manuscript to a SpringerOpen ${ }^{\circ}$ journal and benefit from:}

- Convenient online submission

- Rigorous peer review

- Open access: articles freely available online

- High visibility within the field

- Retaining the copyright to your article

Submit your next manuscript at $\boldsymbol{\nabla}$ springeropen.com 